\begin{tabular}{|c|c|}
\hline ANGIES & Angles \\
\hline $\begin{array}{l}\text { NEW PERSPEETUES } \\
\text { ANGLOPHINE WNTRLD }\end{array}$ & New Perspectives on the Anglophone World \\
\hline & $\begin{array}{l}2 \mid 2016 \\
\text { New Approaches to the Body }\end{array}$ \\
\hline
\end{tabular}

\title{
The Actors' Bodies in TV series LOST
}

\section{Sylvaine Bataille and Sarah Hatchuel}

\section{(2) OpenEdition}

\section{Journals}

\section{Electronic version}

URL: https://journals.openedition.org/angles/1893

DOI: 10.4000/angles. 1893

ISSN: 2274-2042

\section{Publisher}

Société des Anglicistes de l'Enseignement Supérieur

Electronic reference

Sylvaine Bataille and Sarah Hatchuel, "The Actors' Bodies in TV series LOST", Angles [Online], 2 | 2016, Online since 01 April 2016, connection on 08 June 2022. URL: http://journals.openedition.org/angles/ 1893 ; DOI: https://doi.org/10.4000/angles.1893

This text was automatically generated on 8 June 2022.

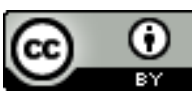

Angles est mise à disposition selon les termes de la Licence Creative Commons Attribution 4.0 International. 


\title{
The Actors' Bodies in TV series LOST
}

\author{
Sylvaine Bataille and Sarah Hatchuel
}

This media file cannot be displayed. Please refer to the online document http:// journals.openedition.org/angles/1893

\section{Transcript:}

1 Au début de Lost, série diffusée sur $A B C$ entre 2004-2010, un avion s'écrase sur une île du Pacifique. Une quarantaine de passagers échappent de peu à la mort. Perdus dans une zone inconnue du globe, ils découvrent que l'île n'est pas inhabitée et que d'étranges phénomènes s'y produisent. Dans ce qui s'apparente d'abord à une Robinsonnade, comment la série a-t-elle pensé et mis en pratique sa relation aux corps des acteurs et actrices? Lost présente deux originalités : adaptation du script aux acteurs dans un premier temps, évolution narrative laissant libre cours à l'évolution du jeu dans un second. La prise de risque au moment du choix des acteurs se trouve atténuée, et même mise à profit, par le caractère foncièrement collaboratif de l'écriture sérielle. En retour, la prolifération narrative, poussée à l'extrême dans Lost, permet aux acteurs d'utiliser leur corps pour explorer toutes les nuances de leur rôle, jamais figé ni monolithique.

2 A travers un casting souple, dynamique et réactif, le récit de Lost s'est retrouvé subordonné à la réalité physique et au charisme de chaque acteur et actrice, choisis pour leurs différentes personnalités et leurs talents spécifiques. Dans la première esquisse du script, Hurley, Sayid, Jin et Sun n'avaient pas été imaginés; d'autres personnages étaient encore très flous : le rôle de Charlie était à l'origine prévu pour être celui d'un vieux rocker has-been, quant à Sawyer, il devait être un arnaqueur sophistiqué du Nord-Est des États-Unis. Plusieurs acteurs qui ont été auditionnés pour le rôle de Sawyer ont finalement eu un rôle " sur mesure » : c'est le cas de Jorge Garcia, pour qui le personnage de Hurley a été expressément créé, c'est aussi le cas de Dominic Monaghan, pour qui le rôle de Charlie a été adapté. L'acteur Josh Holloway, qui a passé toute sa jeunesse en Géorgie, a changé la donne pour le personnage de Sawyer, qui a été transformé en homme du Sud ${ }^{1}$. Les acteurs et leur persona ont ainsi été placés au centre 
du dispositif : ce fut au script de s'adapter à leurs contours, y compris physiques, un mode de casting qui inversait les pratiques habituelles.

3 Les personnages de Lost ont été adaptés à la persona des acteurs, apparue dès les auditions. Cette porosité entre personnage et acteur a été facilitée par le fait que les acteurs étaient, dans leur grande majorité, inconnus du grand public occidental, et n'étaient pas marqués par des rôles importants au cinéma ou à la télévision. Hervé Glevarec, dans son ouvrage sur La Sériephilie, souligne que ce manque de notoriété préalable est un point souvent remarqué et apprécié des amateurs de séries télévisées (Glevarec 2012: 98). Lost a poussé cette logique très loin: non seulement les acteurs n'étaient pas connus et pouvaient créer une identité fictionnelle forte, crédible et quasi autonome pour leur personnage, mais le rôle a été construit autour de et pour leur personnalité, facilitant ainsi leur entrée dans une identité moins «autre " qu’à l'accoutumée, entre rôle fictif et personnalité d'origine ${ }^{2}$.

4 Ce mélange des notions de rôle (qui s'insère normalement dans une structure dramaturgique), de personnage (qui s'insère dans une structure fictionnelle) et d'identité (en lien avec une supposée personnalité "réelle») est à l'origine de remarques intéressantes sur les forums de discussion. Sous la vidéo Youtube de l'audition de Josh Holloway ${ }^{3}$, on peut lire "Sawyer does a GREAT Josh Holloway », dans une inversion révélatrice et métadramatique des rôles. Sawyer, personnalité cynique aux cheveux longs, devient ici l'individu réel (tant les six saisons de Lost lui ont apporté substance et complexité), qui jouerait à être un Josh Holloway pré-Lost, aux cheveux courts et en brosse, que les amateurs connaissent beaucoup moins bien que le personnage de fiction. Cette persona du personnage affecte le comédien, sa carrière, sa réception par le public, et notamment les publicités tournées par les acteurs.

Dans l'épisode 16 de la Saison 2, diffusé le 22 mars 2006 aux États-Unis, Jack, interprété par Matthew Fox, sort de la douche et est surpris de trouver Locke (joué par Terry O'Quinn) en train d'occuper la salle de bain et de se raser devant le miroir. Locke explique son intrusion en lui disant que «la vapeur permet d'ouvrir les pores $»^{4}$. Ce contexte très métrosexuel où deux hommes prennent soin, en même temps, de leur petite personne et se donnent des conseils, s'est reflété dans la publicité tournée par Matthew Fox pour Men Expert, crème hydratante de L'Oréal, publicité diffusée internationalement en 2007, juste un an après l'épisode en question ${ }^{5}$. En France, la Saison 2, programmée un an après la diffusion américaine, a même été sponsorisée par Men Expert de L'Oréal ${ }^{6}$ : la publicité a donc été vue avant l'épisode de la douche et a contribué à construire rapidement Jack en personnage anti-macho - sensible, responsable et prévenant.

6 En janvier 2010, Josh Holloway tourne à Hawaï (lieu où la série est également filmée) la publicité pour Cool Water de Davidoff ${ }^{7}$ : La publicité est une reprise de deux séquences de Lost : dans la première, Sawyer sort de l'eau devant Kate dans l'épisode 8 de la Saison 1 (diffusé le 10 novembre 2004) ${ }^{8}$. La deuxième séquence qui a inspiré la publicité est celle où Sawyer regagne la plage, sous les yeux de Juliet, après avoir sauté de l'hélicoptère en pleine mer dans l'épisode 14 de la Saison 4 (diffusé le 29 mai 2008). Mais la publicité de Cool Water n'est pas une simple reprise. Elle a aussi inspiré une séquence du dernier épisode de la série diffusé le 23 mai 2010 où Sawyer saute d'une falaise en pleine mer pour rejoindre l'avion de Frank Lapidus9. Les productions diégétiques et extra-diégétiques sont ainsi entrées en interaction et en résonnance. Le slogan de Davidoff, "The power of cool», s'appuie sur l'identité fictionnelle de Sawyer, 
directement influencée par la persona d'Holloway, alliant cynisme débonnaire, détachement amusé et conscience réjouie et assumée de son pouvoir de séduction. Si la série a adapté ses personnages aux acteurs qui les jouaient, le script a ensuite permis à ces mêmes acteurs d'évoluer. Lorsque Sawyer vit une histoire d'amour avec Juliet, dans le passé de l'île en Saison 5, c'est même son regard qui en vient à changer, empreint d'une douceur que l'on n'avait encore jamais vue.

7 Lost développe une narration complexe en intégrant flashbacks, flashforwards, récits parallèles et voyages dans le temps qui viennent multiplier les trames narratives. Si le récit linéaire permet l'évolution du jeu des acteurs sur le long terme, les récits connexes leur offrent la possibilité d'explorer différentes facettes de leur rôle, lorsqu'ils sont amenés à jouer leur personnage à un moment différent de sa vie, dans un contexte tout autre que celui de l'île.

8 La narration de Lost utilise le corps des acteurs, soit en modifiant leur apparence, à l'aide de perruques ou de barbes postiches, soit en exploitant des particularités physiques. Ainsi les tatouages de l'acteur Matthew Fox $^{10}$ ont inspiré un récit en flashback qui révèle dans quelles circonstances Jack a été tatoué (3.9). Dès la première saison (1.6), Kate fait remarquer que les tatouages sur le bras de Jack ne correspondent pas à l'image qu'elle se fait d'un neurochirurgien. Cette remarque peut être interprétée comme un commentaire auto-référenciel, une forme d'ironie malicieuse de la série sur son propre casting: en pointant le décalage entre le corps du personnage et son rôle social, le script semble pointer du doigt ce qui pourrait paraitre à première vue comme une inadéquation entre le corps de Matthew Fox et le personnage pour lequel il a été choisi, comme une erreur de casting. Bien sûr, les multiples récits connexes consacrés à Jack viennent fournir la preuve, s'il en était besoin, que Matthew Fox était le bon choix, et que ses tatouages, loin de décrédibiliser son rôle, contribuent à l'épaisseur et à la complexité du personnage.

9 Le corps en pleine croissance du jeune acteur Malcolm David Kelley, qui jouait un garçon de 10 ans, Walt, dans la première saison a posé davantage de problèmes à la production, car il n'y a pas d'homochronie entre le récit des événements sur l'île et la diffusion de la série : le temps qui s'écoule sur l'île au fil de chaque saison ne dure que quelques dizaines de jours pour les personnages. L'acteur a donc grandi trop vite par rapport à son personnage et n'a fait que de brèves apparitions après la saison 1 , soit dans des plans larges dans lesquels son apparence était modifiée numériquement pour qu'il semble plus jeune ${ }^{11}$, soit dans des scènes censées se dérouler plusieurs années après le crash de l'avion.

10 La narration a pu en revanche exploiter le passage du temps sur les corps des acteurs adultes. Différentes apparences physiques ont été créées pour marquer différentes périodes dans la vie des personnages, et parfois aussi pour symboliser divers états psychiques. Lorsque Claire réapparaît après trois ans d'absence dans la diégèse, la chevelure ébouriffée de l'actrice Emilie de Ravin signale immédiatement que le personnage a radicalement changé, et suggère qu'elle a vécu dans un environnement sauvage, à l'écart des micro-sociétés qui peuplent l'île. Le désordre des cheveux de l'actrice symbolise aussi le désordre mental du personnage, désormais « infectée » par une mystérieuse maladie.

11 Les flashbacks, les flashforwards et les flashsideways constituent d'épisode en épisode les pièces d'un puzzle narratif : la présence ou non de certaines particularités physiques fournit un indice aux spectateurs, qui, en faisant appel à leur mémoire ou à leur sens 
logique, l'associent à une période de la vie du personnage et peuvent ainsi situer temporellement les événements racontés. Ou du moins, ils croient pouvoir le faire, car la série multiplie les surprises et les fausses pistes. Par exemple, deux éléments dans l'apparence de Terry O'Quinn permettent de distinguer différentes périodes de la vie de Locke : le fait qu'il se déplace en fauteuil roulant ou non indique si la scène se passe avant ou après son accident ; la présence ou non de cheveux sur son crâne, est bien sûr un indicateur de son âge. Le flashback consacré à Locke dans l'épisode 13 de la Saison 3 joue sur les conjectures des téléspectateurs en présentant tout d'abord Locke assis dans un bureau devant une fonctionnaire qu'il est venu voir au sujet d'une allocation d'invalidité. Vu le contexte, nous avons tout lieu de penser qu'il est déjà handicapé. Mais la scène déjoue nos attentes dans un clin d'œil au tout premier flashback consacré à Locke. Celle-ci mettait en scène une situation assez similaire d'échange autour d'un bureau pour nous révéler, au moment où Locke quitte la pièce, qu'il était en fait assis sur un fauteuil roulant. Cette fois-ci Locke se lève et on saisit finalement que l'invalidité dont il était question était en fait son état dépressif. Les modifications apportées à l'apparence physique des acteurs sont ainsi tout autant des repères temporels que des éléments de surprise ou de brouillage.

Un autre exemple est la barbe de Matthew Fox dans les flashforwards consacrés à Jack, qui apparaît plus ou moins fournie dans le récit proleptique et contribue à nos interrogations concernant le moment où sont censés se dérouler les événements : nous n'avons encore jamais vu le personnage sous cet aspect, que ce soit sur l'île (où il parvient apparemment à se raser) ou dans les flashbacks, et nous ne pouvons donc pas relier son apparence avec une période déjà identifiée. Ce que nous pouvons saisir, en revanche, c'est la dimension symbolique de cet excès de pilosité, le signe du désordre qui règne dans la vie de ce Jack suicidaire. Ce n'est qu'à la toute fin du flashforward que nous pouvons identifier ce récit comme une prolepse, lorsque Jack retrouve Kate hors de l'île et tente de la convaincre qu'ils doivent y retourner.

C'est dans la dernière saison que la diégétisation de cette tension entre rôles sur mesure et rôles évolutifs prend toute sa force: à travers le procédé narratif du flashsideways et ses effets de miroir, qui ramène le script à son point de départ tout en offrant une vision alternative des rôles, la série reconfigure le travail des acteurs, donnant $\mathrm{du}$ « jeu » au jeu.

14 À la fin de la Saison 5, les personnages propulsés dans les années 70 tentent de changer le cours du temps en faisant exploser l'île grâce à une bombe à hydrogène. Si l'île n'existe plus, ses perturbations magnétiques ne pourront plus affecter le vol 815 en 2004, l'avion ne s'écrasera plus et la vie des personnages pourra suivre un autre chemin, ouvrant donc une autre ligne temporelle à partir de la disparition de l'île. C'est cette idée qu'explorent, à première vue, les flash-sideways dans la sixième et dernière saison, où le vol Oceanic 815 ne s'écrase pas sur l'île mais se pose sans problème à l'aéroport de Los Angeles. Ces séquences exposent la vie des personnages si l'île n'existait plus à partir de 1977. Les flash-sideways transforment la réalité en l'un des innombrables mondes possibles et créent le spectre d'un univers qui « aurait pu être " et qui ne cesse de hanter tous les autres. Les personnages ont vécu d'autres expériences, ont suivi une autre voie. Ces séquences parallèles donnent ainsi l'occasion aux acteurs de montrer une autre facette de leur jeu, comme s'ils préparaient leur reconversion dans d'autres rôles après la fin de la série et intégraient à l'histoire des tests de casting pour d'éventuels producteurs. 


\section{BIBLIOGRAPHY}

\section{Credits}

Video essay: The actors' bodies in TV series LOST

Text: Sylvaine Bataille, (ERIAC, Université de Rouen) and Sarah Hatchuel (GRIC, Université du Havre) in the context of the GUEST-Normandie research programme (Groupe Universitaire d'Etudes sur les Séries Télévisées basé en Normandie). This essay was first presented during the "Actors in TV series" workshop organized by Christian Viviani (LASLAR) at the University of Caen (2 February 2013) and the "Bodies in TV series" seminar organized by Florence Cabaret and Odette Louiset (ERIAC) at the University of Rouen (5 June 2015).

Voice, Editor and Subtitles: Sarah Hatchuel

Music: Michael Giacchino

Copyrights (pictures and excerpts): ABC/Bad Robot. All rights reserved. 
December 2015, for ANGLES.

\section{Selective bibliography}

Baridon, Laurent, and Martial Guédron. Corps et arts, physionomies et physiologies dans les arts visuels. Paris : L'Harmattan, 1999.

Burdeau, Emmanuel, ed. Tours de rôles - acteurs et actrices d'un film à l'autre. Saint-Sulpice-surLoire : Association des cinémas de l'Ouest pour la recherche, 2007.

Butler, Judith. Bodies That Matter: On the Discursive Limits of "Sex". Routledge: New York, 1993.

Cohan, Steven, and Ina Rae Hark, eds. Screening the Male: Exploring Masculinities in Hollywood Cinema. London: Routledge, 1993.

Devlin, William J. "Imaginary Peanut Butter: The Puzzles of Time Travel in Lost." In The Ultimate Lost and Philosophy: Think Together, Die Alone. Ed. Sharon Kaye. Hoboken : John Wiley \& Sons, 2011. $32-46$.

Dyer, Richard. Stars. London: BFI, 1979.

Damour, Christophe, Christian Gutleben, Hélène Valmary and Christian Viviani, eds. Généalogies de l'acteur au cinéma : Échos, influences, migrations. Cycnos, 27.2 (2011). http://revel.unice.fr/cycnos/ index.html?id=6755

Dureau, Christian. Acteurs de séries et feuilletons. Le Dictionnaire. Paris: Didier Carpentier, 2008.

Glevarec, Hervé. La sériephilie : Sociologie d'un attachement culturel. Paris: Ellipses, 2012.

Hatchuel, Sarah. LOST : Fiction vitale. Paris: PUF, 2013.

Sepulchre, Sarah, and Eric Maigret, Eds. Décoder les séries télévisées. Brussels: De Boeck, 2011.

Thiellement, Pacôme. Les Mêmes yeux que LOST. Paris: Léo Scheer, 2011.

Viviani, Christian. Le Magique et le Vrai : L'acteur de cinéma, sujet et objet. Aix-en-Provence : Rouge Profond, 2015

\section{NOTES}

1. Selon Lindelof, « He was not at all that guy but he was so watchable, we were like 'OK, forget that he is a proto-suit-wearing sleek guy, this guy is more of a fearo, Southern, equally intelligent but a different kind of guy entirely, so let's rewrite the character around him ».

2. Glevarec parle du «statut entre-deux de l'acteur de série, incarnation d'une identité fictionnelle plutôt que d'un rôle" (Glevarec 2012: 100).

3. Voir https://www.youtube.com/watch?v=JQAuzBEple8.

4. Voir la séquence ici : https://www.youtube.com/watch?v=7iADoSDN10c.

5. Voir la publicité en versions anglaise et française: https://www.youtube.com/watch? $\mathrm{v}=$ pRx8NoN2D5s et https://www.youtube.com/watch?v=ooAvXH_3XQ0.

6. Voir la vidéo promotionnelle ici : https://www.youtube.com/watch?v=Au5gnixzOTo.

7. Voir l'article de Style News du 15 janvier 2010 : https://people.com/style/exclusive-behind-thescenes-at-josh-holloways-sexy-cool-water-shoot/. La publicité de Cool Water peut être vue ici : https://www.youtube.com/watch?v=hB4wiadGLLs.

8. https://www.youtube.com/watch?v=c9EOQmRrt2Q.

9. https://www.youtube.com/watch?v=K62-P2IdrLI. 
10. L'acteur avait des tatouages avant de jouer dans la série (voir par exemple : http:// www.telegraph.co.uk/culture/tvandradio/7187894/Matthew-Fox-interview.html encore « Season 2 Podcast - 19 » : http://www.youtube.com/watch?v=-YLu26yjvU8.

11. Voir à ce propos le commentaire audio de S4E13 et S4E14 sur le DVD de la saison 4, retranscrit sur Lospedia. «Carlton Cuse : It's something we struggled with the course of the show. How do we use Walt, when in fact the actor has grown up and gotten older? When in fact, in island time, each season maybe only lasts an average of, like, you know, or 30 days. So...

Damon Lindelof: People asked, how are you gonna deal with the fact that he's growing older? Our solution season one was to have him be abducted by the Others. And at the end of season two, he looks older, but you don't see enough of him as he goes sailing off with Michael. And then we basically sort of had to bench Walt, you know, until now. You do see him briefly at the beginning of season four, about midway through Meet Kevin Johnson, he looks up at the window, and you see a younger version of Walt. That's supposed to be taking place in 4. And that's an effects shot. Mitch Suskin basically lifted a shot of Malcolm back from season one, and put him in that window.

Carlton Cuse : But here now you actually get to see Walt at his actual age. It really worked well for the scene. We love the poignancy of Walt in this scene, asking about the fate of his father."

\section{ABSTRACTS}

This video essay explores the way TV series LOST (ABC, 2004-2010) dramatized its relation to the actors' bodies and included a reflection on the actors' performances within the story itself.

Cet essai-vidéo se propose d'analyser la manière dont la série LOST (ABC, 2004-2010) a pensé et mis en pratique sa relation au corps des acteurs et actrices et de montrer comment le programme a diégétisé sa relation au jeu des comédiens.

\section{INDEX}

Keywords: Lost, TV series, body, actor, audition, commercials, Fox Matthew, Holloway Josh Mots-clés: Lost, série télévisée, corps, acteur, audition, publicité, Fox Matthew, Holloway Josh

\section{AUTHORS}

\section{SYLVAINE BATAILLE}

Sylvaine Bataille is a Lecturer in the English department at the University of Rouen, a member of ERIAC and the head of GUEST-Normandie, a research program on TV series. She works on the questions of appropriation, adaptation, translation and reference in $16^{\text {th }}$ and $17^{\text {th }}$ century English literature and in today's popular culture, with a focus on screen adaptations of Shakespeare's plays and drama TV series. Recent publications include: "Battlestar Galactica et l'héritage grécolatin", in L'Antiquité dans l'imaginaire contemporain, Fantasy, science-fiction, fantastique, dir. Mélanie Bost-Fiévet et Sandra Provini, Paris, Classiques Garnier (2014_and “"Domestic fury and 
fierce civil strife/Shall cumber all the parts of Italy' : la guerre civile à l'écran dans Julius Caesar de Joseph L. Mankiewicz", in La Guerre civile: représentations, idéalisations, identifications, Cahiers de l'ERIAC $n^{\circ}$ 6, dir. Claire Gheeraert-Graffeuille et Emmanuel Dupraz (2014). http:// eriac.univ-rouen.fr/author/sylvaine-bataille/. Contact: sylvaine.brennetot [at] univ-rouen.fr

\section{SARAH HATCHUEL}

Sarah Hatchuel is Professor of English Literature and Film at the University of Le Havre (France), President of the Société Française Shakespeare and head of the 'Groupe de recherché Identités et Cultures'. She has written extensively on adaptations of Shakespeare's plays (Shakespeare and the Cleopatra/Caesar Intertext: Sequel, Conflation, Remake, Fairleigh Dickinson University Press, 2011; Shakespeare, from Stage to Screen, Cambridge University Press, 2004; A Companion to the Shakespearean Films of Kenneth Branagh, Blizzard Publishing, 2000) and on TV series (Lost: Fiction vitale, PUF, 2013; Rêves et series américaines: la fabrique d'autres mondes, Rouge Profond, 2015). She is the general editor of the CUP Shakespeare on Screen collection (with Nathalie Vienne-Guerrin) and of the online journal TV/Series (with Ariane Hudelet). Contact: sarah.hatchuel [at] univ-lehavre.fr 